\title{
MAXIMAL CHAINS OF PRIME IDEALS IN INTEGRAL EXTENSION DOMAINS. II
}

BY

\author{
L. J. RATLIFF, JR.( $\left.{ }^{\mathbf{1}}\right)$
}

\begin{abstract}
Four related subjects are investigated: (1) If $(L, N)$ is a locality over a local domain $(R, M)$ such that $N \cap R=M$, and if there exists an integral extension domain of $L$ which has a maximal chain of prime ideals of length $n$ (for short, a mcpil $n$ ), then there exists an integral extension domain of $R$ which has a mcpil $n-\operatorname{trd} L / R+\operatorname{trd}(L / N) /(R / M)$. A refinement of the altitude inequality follows from this. (2) A condition for the converse of (1) to hold is given. (3) The class of local domains $R$ such that there exists an integral extension domain of $R$ which has a mcpil $n$ if and only if there exists a mcpil $n$ in $R$ is studied. (4) Two new equivalences for the existence of mcpil $\boldsymbol{n}$ in an integral extension domain of a local domain are given.
\end{abstract}

1. Introduction. Throughout this introduction let $(R, M)$ be a local domain and let $c(R)=\{n$; there exists an integral extension domain of $R$ which has a maximal chain of prime ideals of length $n$ \}.

This paper is concerned with the relationship between $c(R)$ and $c(L)$, where $(L, N)$ is a locality over $R$ such that $N \cap R=M$ (that is, $L$ is a local quotient ring of a finitely generated integral domain over $R$ ). The exact relationship is found, and it turns out to be very closely related to the altitude formula and the altitude inequality (see (2.1) for the definitions). (In deriving the relationship (and in all the results in this paper) we heavily rely on the main theorem in [17]; in fact, such heavy use of this result is made that it is summarized in (a)-(f) at the end of this introduction.) We look at some consequences of the relationship, and consider some related subjects. We now give some specific information on the contents of this paper.

In $\S 2$ it is proved that if $(L, N)$ is a locality over $R$ such that $N \cap R=M$, and if $n \in c(L)$, then $n-\operatorname{trd} L / R+\operatorname{trd}(L / N) /(R / M) \in c(R)$ (2.5). An immed-

Received by the editors May 28, 1975.

AMS (MOS) subject classifications (1970). Primary 13A15, 13B20, 13C15; Secondary $13 \mathrm{B25}, 13 \mathrm{~B} 99$.

Key words and phrases. Algebraic extension, altitude formula, altitude inequality, analytically independent, catenary ring, chain condition for prime ideals, chain conjecture, completion of local ring, first chain condition, form ring, $H$ conjecture, $H_{i}$-ring, Henselian local ring, integral extension, local ring, locality, maximal chain of prime ideals, Noetherian ring, quadratic transformation, quasi-unmixed local ring, Rees ring, second chain condition, subspace, transcendental extension ring, unmixed local ring, upper conjecture.

(1) Research supported in part by NSF-MCS 76-06009. 
iate corollary of this is the following refinement to the altitude inequality: With $(R, M)$ and $(L, N)$ as above, there exists $m \in c(R)$ such that altitude $L+$ $\operatorname{trd}(L / N) /(R / M)=m+\operatorname{trd} L / R \leqslant$ altitude $R+\operatorname{trd} L / R$ (2.6.2). Then some further corollaries of (2.5) are given in (2.7)-(2.11).

In $\$ 3$ a condition for the converse of (2.5) to hold is given in (3.1), and then some corollaries of (3.1) are given. For example, if $R=R(R, I)$ is the Rees ring (3.4) of $R$ with respect to an ideal $I$ in $R$ and $M$ is the maximal homogeneous ideal in $R$, then $n \in c(R)$ if and only if $n+1 \in c\left(R_{u}\right)$ (3.6). (3.7) shows that for certain $M$-primary ideals $B$ in $R, n+1 \in c\left(R_{M}\right)$ if and only if there exists a prime ideal $p$ in $R_{M}$ such that height $p=1$ and depth $p=n$. This is a very important property, as is noted in the last paragraph of $\S 4$.

In $\S 4$ we consider the class $C$ of local domains $R$ such that $n \in c(R)$ if and only if there exists a maximal chain of prime ideals of length $n$ in $R$. In (4.1) we show that the following rings are in $C: R[X]_{(M, X)}$, for all local domains $R$; all Henselian local domains; and, all local domains wihch satisfy the s.c.c. (2.1.3). Certain other properties of $C$ are given in (4.1). (4.2) gives some properties of the rings which are in $C$. Some questions about $C$ are asked in (4.5)-(4.7), and one of these is partially answered in (4.8.2) by showing, in particular, that if $R$ $\in C$ is integrally closed and $N$ is a maximal ideal in a finite integral extension domain $S$ of $R$, then $S_{N} \in C$ and $c(R)=c\left(S_{N}\right)$. Also, some equivalences of the upper conjecture are given which relate it to the class $C(4.10 .3)$.

In (5.2) we give two more equivalences of the six equivalent conditions given in $[17,(2.14)]$ (see (a)-(f) below) for a local domain, and show that these new conditions are equivalent, in the local ring case, to two of the conditions in [17]. One of these new equivalent conditions is that, for the domain case, $n \in$ $c(R)$ if and only if there exists a height $n-1$ depth one prime ideal in the Henselization of $R$. Some consequences of (5.2) relating to $H_{i}$-rings (5.3) are given in (5.4) and (5.6), and then we close this section with two propositions (5.9) and (5.10) which characterize all local domains $R$ such that card $c(R) \leqslant 2$. (Nagata's examples have this property.)

In $\$ 6$ some results are given showing the existence of certain rings contained in the quotient field $F$ of $R$ which have certain types of maximal chains of prime ideals. (6.1) shows that if $n \in c(R)$, then there exists a locality $L=$ $R[d]_{(M, d)} \subseteq F$ such that there exists a maximal chain of prime ideals of length $n$ in $L$. Also, there exists a maximal ideal $N$ in a finitely generated ring $A \subseteq F$ over $R$ such that $N \cap R=M$ and height $N=n$ (6.3). Finally, (6.7) shows that if $m>1$ is the smallest element in $c(R)$, then there exists a quadratic transformation $L$ of $R$ such that $n \in c(R)$ if and only if $n-m+1 \in c(L)$.

Throughout, we show that certain known results easily follow from the new material in this paper. Also, a number of questions are asked, and frequent re- 
marks are interspersed which relate the new results to other results in this paper and in other papers.

Since we will frequently need to refer to the main result in [17], we close this introduction by stating it. For typographic reasons, we index the separate parts by a single letter.

Let $(R, M)$ be a local domain, let $k$ be a positive integer, let $Q$ be a prime ideal in $R_{k}=R\left[X_{1}, \ldots, X_{k}\right]$ such that $M R_{k} \subset Q$, and let $S=\left(R_{k}\right)_{Q}$. Then the following statements are equivalent:

(a) There exists an integral extension domain of $R$ which has a mcpil $n$ (see (2.2) below).

(b) There exists a minimal prime ideal $z$ in the completion of $R$ such that depth $z=n$.

(c) There exists a minimal prime ideal $w$ in the completion of $S$ such that depth $w=n+k-\operatorname{depth} Q$.

(d) There exists an integral extension domain of $S$ which has a mcpil $n+$ $k$ - depth $Q$.

(e) There exists a mcpil $n+k-\operatorname{depth} Q$ in $S$.

(f) There exists a mcpil $n+1$ in $R\left[X_{1}\right]_{\left(M, X_{1}\right)}$.

2. On the altitude inequality. All rings in this paper are assumed to be commutative rings with an identity element. The undefined terminology is the same as that in [6]. We mention, in particular, that $A \subset B$ denotes proper containment.

The following terminology will be frequently used in what follows.

(2.1) Definitions. Let $A$ be an integral domain, and let $a=$ altitude $A<\infty$.

(2.1.1) $A$ satisfies the first chain condition for prime ideals (f.c.c.) in case every maximal chain of prime ideals in $A$ has length $=a$.

(2.1.2) $A$ is catenary in case, for each pair of prime ideals $P \subset Q$ in $A$, $(A / P)_{Q / P}$ satisfies the f.c.c.

(2.1.3) $A$ satisfies the second chain condition for prime ideals (s.c.c.) in case every integral extension domain of $A$ satisfies the f.c.c.

(2.1.4) $A$ satisfies the chain condition for prime ideals (c.c.) in case, for each pair of prime ideals $P \subset Q$ in $A,(A / P)_{Q / P}$ satisfies the s.c.c.

(2.1.5) $A$ is quasi-unmixed (resp., unmixed) in case $A$ is semilocal and every minimal (resp., every) prime divisor of zero in the completion of $A$ has depth $=a$.

(2.1.6) $A$ satisfies the altitude formula in case, for each finitely generated 
integral domain $B$ over $A$ and for each prime ideal $P$ in $B$, altitude $B_{P}+$ $\operatorname{trd}(B / P) /(A /(P \cap A))=$ altitude $A_{P \cap A}+\operatorname{trd} B / A$, where $\operatorname{trd} C / D$ denotes the transcendence degree of the quotient field of the integral domain $C$ over the quotient field of its subdomain $D$.

(2.1.7) $A$ satisfies the altitude inequality in case, for each $B$ and $P$ as in (2.1.6), altitude $B_{P}+\operatorname{trd}(B / P) /(A /(P \cap A)) \leqslant$ altitude $A_{P \cap A}+\operatorname{trd} B / A$.

It is known [21, Proposition 2, p. 326] that a Noetherian domain satisfies the altitude inequality. This fact will often be used below.

We also define mcpil and $W(k, P)$ as in [17]. Specifically, we need the following two definitions.

(2.2) Definition. It will be said that a ring $A$ has a mcpil $n$ in case there exists a maximal chain of prime ideals of length $n$ in $A$ (that is, a chain of prime ideals $p_{0} \subset p_{1} \subset \cdots \subset p_{n}$ such that $p_{0}$ is minimal, $p_{n}$ is maximal, and height $p_{i} / p_{i-1}=1(i=1, \ldots, n)$.

(2.3) Definition. Let $A$ be an integral domain.

(2.3.1) $W(k, P)=\left\{n\right.$; there exists a mcpil $n$ in $\left.\left(A_{k}\right)_{P}\right\}$, where $k$ is a nonnegative integer and $P$ is a prime ideal in $A_{k}=A\left[X_{1}, \ldots, X_{k}\right]\left(A_{0}=A\right)$.

(2.3.2) $c(A)=\{n$; there exists an integral extension domain of $A$ which has a mcpil $n$ \}.

(2.4) Remarks. (2.4.1) Clearly, altitude $A$ is the largest integer in $c(A)$.

(2.4.2) It is clear from the definitions that $A$ satisfies the s.c.c. (2.1.3) if and only if $c(A)=$ altitude $A$ \}.

(2.4.3) A local domain $R$ is quasi-unmixed (2.1.5) if and only if $c(R)=$ \{ altitude $R$ \}, since, by (a) $\Longleftrightarrow(\mathrm{b}), c(R)=\{$ depth $z ; z$ is a minimal prime ideal in the completion of $R$ \}.

(2.4.4) If $(R, M)$ is a local domain, then $W\left(1,\left(M, X_{1}\right)\right)=\{n+1 ; n \in$ $c(R)\}$, by (a) $\Longleftrightarrow$ (f).

The following theorem will allow us to give a refinement to the altitude inequality, as will be shown in (2.6.2) below. To prove (2.5), the following known result is needed: A transcendental extension domain of a Noetherian domain $\boldsymbol{A}$ satisfies the altitude formula relative to $A$ [21, Proposition 2, p. 326].

(2.5) THEOREM. Let $(R, M)$ and $(L, N)$ be local domains such that $L$ is a locality over $R$ and $N \cap R=M$. If $n \in c(L)$, then $n-\operatorname{trd} L / R+\operatorname{trd}(L / N) /(R / M)$ $\in c(R)$.

Proof. Let $L=A_{P}$, where $A=R\left[d_{1}, \ldots, d_{m}\right]$, let $K$ be the kernel of the natural homomorphism from $R_{m}=R\left[X_{1}, \ldots, X_{m}\right]$ onto $A$, and let $P^{*}$ be the preimage of $P$ in $R_{m}$. Let $t=\operatorname{trd} L / R$ and $t^{\prime}=\operatorname{trd}(A / P) /(R / M)=($ since $R / M$ 
is a field) altitude $A / P=\operatorname{depth} P=\operatorname{depth} P^{*} / K=\operatorname{depth} P^{*}$. Also, by the altitude formula for $R_{m}$ over $R$ [21, Proposition 2, p. 326], height $K+t=$ height $K \cap R+m$; that is, height $K=m-t$.

Now, if $n \in c(L)$, then there exists an integral extension domain, say $B$, of $R_{m}$ which has a saturated chain of prime ideals of length $n$, say $p_{0} \subset \cdots \subset p_{n}$ such that $p_{0} \cap R_{m}=K$ and $p_{n} \cap R_{m}=P^{*}$. Then, since $\left(R_{m}\right)_{K}$ satisfies the s.c.c. (since it is a regular local ring (since $K \cap R=(0)$ )), height $p_{0}=$ height $K=$ $m-t$. Therefore, since depth $p_{n}=$ depth $P^{*}=t^{\prime}$, this chain can be extended to a mcpil $n+t^{\prime}+m-t$ in $B$, say (0) $\subset \cdots \subset p_{0} \subset \cdots \subset p_{n} \subset \cdots \subset q$ (height $p_{0}=m-t$ and height $\left.q / p_{n}=t^{\prime}\right)$. Let $Q=q \cap R_{m}$. Then this chain extends to a mcpil $n+t^{\prime}+m-t$ in $B_{\left(R_{m}-Q\right)}$, and $B_{\left(R_{m}-Q\right)}$ is integral over $\left(R_{m}\right)_{Q}$. Hence, since $M R_{m} \subset Q$ (since $Q$ is maximal and $Q \cap R=M$ ), it follows from (a) $\Longleftrightarrow$ (d) that there exists an integral extension domain of $R$ which has a mcpil $n+t^{\prime}$ $-t$, that is $n+t^{\prime}-t \in c(R)$. Q.E.D.

The converse of $(2.5)$ is not true, in general. For example, let $(R, M)$ be as in [6, Example 2, pp. 203-205] in the case $r>0$ and $m>0$. Then altitude $R$ $=r+m+1=$ (say) $a$, and the integral closure $R^{\prime}$ of $R$ is a finite $R$-algebra and has a maximal ideal, say $Q$, such that height $Q=m+1<a$. Let $L=\left(R^{\prime}\right)_{Q}$. Then clearly $a \in c(R)$, but $a \notin c(L)$ (since altitude $L<a$ ).

However, the converse of (2.5) is true in many important cases, as will be shown in (3.1) below.

(2.6.2) could be called a refinement to the altitude inequality (2.1.7).

(2.6) CoRollary. Let $(R, M)$ and $(L, N)$ be as in (2.5). Then the following statements hold:

(2.6.1) There exists an order-preserving injection $f: c(L) \rightarrow c(R)$ given by $f(n)=n-\operatorname{trd} L / R+\operatorname{trd}(L / N) /(R / M)$, for $n \in c(L)$.

(2.6.2) There exists $m \in \mathrm{c}(R)$ such that altitude $L+\operatorname{trd}(L / N) /(R / M)=$ $m+\operatorname{trd} L / R \leqslant$ altitude $R+\operatorname{trd} L / R$.

ProOF. (2.6.1) The existence of $f$ is clear by (2.5).

(2.6.2) Since altitude $L \in c(L)$ and altitude $R$ is the largest element in $c(R)$ (2.4.1), (2.6.2) follows by letting $m=f$ (altitude $L$ ). Q.E.D.

The existence of the map $f$ in (2.6.1) can be used to give an easy proof of some well-known results. For example, see (2.7.2) and (2.7.3).

(2.7) REMARK. Let $(R, M),(L, N)$, and $f: c(L) \rightarrow c(R)$ be as in (2.6). Then the following statements hold:

(2.7.1) If $a=$ altitude $R \in \operatorname{Im}(f)$ (in particular, if $f$ is a bijection, then

$$
\text { altitude } L+\operatorname{trd}(L / N) /(R / M)=\text { altitude } R+\operatorname{trd} L / R \text {. }
$$

(2.7.2) If $R$ satisfies the s.c.c., then $L$ satisfies the s.c.c. and (*) holds. 
(2.7.3) If altitude $R \leqslant 1$, then $R$ satisfies the s.c.c.; hence $L$ satisfies the s.c.c.

PROOF. (2.7.1) If $a \in \operatorname{Im}(f)$, then there exists $n \in c(L)$ such that $f(n)$ $=n-\operatorname{trd} L / R+\operatorname{trd}(L / N) /(R / M)$. Hence, since by (2.4.1), altitude $L$ and $a$ are the largest elements in $c(L)$ and $c(R)$, respectively, and since $f$ preserves order, the conclusion follows.

(2.7.2) If $R$ satisfies the s.c.c., then $c(R)$ is a one-point set (2.4.2); hence $c(L)$ is a one-point set (since $f$ is injective); hence $L$ satisfies the s.c.c. (2.4.2). Further, $a \in \operatorname{Im}(f)$; hence (*) holds, by (2.7.1).

(2.7.3) If altitude $R \leqslant 1$, then $c(R)$ is a one-point set, hence $R$ satisfies the s.c.c. (2.4.2), and so $L$ satisfies the s.c.c. (2.7.2). Q.E.D.

(2.8) COROLLARY. Let $A$ be a finitely-generated integral domain over a local domain $(R, M)$. Then the following statements hold:

(2.8.1) For each prime ideal $P$ in $A$ such that $P \cap R=M$, height $P+$ $\operatorname{trd}(A / P) /(R / M) \in\{m+t: m \in c(R), t=\operatorname{trd} A / R\}$.

(2.8.2) For each maximal ideal $N$ in $A$ such that $N \cap R=M$, height $N \in$ $\{m+t ; m \in c(R), t=\operatorname{trd} A / R\}$.

Proof. (2.8.1) is clear by (2.6.2).

(2.8.2) follows from (2.8.1), since the field $A / N$ is a finitely-generated integral domain over the field $R / M$; hence $\operatorname{trd}(A / N) /(R / M)=0$. Q.E.D.

With (2.8.2) in mind, it will be shown in (6.3) below that, for each $m \in$ $c(R)$, there exists a locality $(L, N)$ over $R$ such that $N \cap R=M, L \subseteq F=$ the quotient field of $R$, and height $N=m$.

The following two corollaries to (2.5) are concerned with algebraic extension domains.

(2.9) COROllary. Let $(R, M)$ be a local domain, and let $A$ be a finitelygenerated algebraic extension domain of $R$. Then, for each maximal ideal $N$ in $A$ such that $N \cap R=M, c\left(A_{N}\right) \subseteq c(R)$.

Proof. If $N$ is a maximal ideal in $A$ such that $N \cap R=M$, then $\operatorname{trd}(A / N) /(R / M)=0$. Therefore the conclusion follows from (2.5). Q.E.D.

(2.10) CoRollary. Let $b_{0}, b_{1}, \ldots, b_{m}$ be analytically independent elements in a local domain $(R, M)$, and let $A=R\left[b_{1} / b_{0}, \ldots, b_{m} / b_{0}\right]$. If $n \in$ $c\left(A_{M A}\right)$, then $n+m \in c(R)$.

Proof. $M A$ is a prime ideal in $A$ and the residue classes modulo $M A$ of the $b_{i} / b_{0}$ are algebraically independent over $R / M[11$, Lemma 4.3]; hence the conclusion follows from (2.5). Q.E.D.

The converse of (2.10) is true in some cases, as will be shown in (6.7).

It is known [9, Corollary 2.5] that a local domain $(L, N)$ which is a locality over a quasi-unmixed local domain $(R, M)$ is quasi-unmixed. (2.11.1) generalizes this. 
(2.11) REMARK. Let $(R, M)$ and $(L, N)$ be as in (2.5), and let $L^{*}$ and $R^{*}$ be the completions of $L$ and $R$, respectively. Then the following statements hold:

(2.11.1) If $w$ is a minimal prime ideal in $L^{*}$, then depth $w \in\{$ depth $z+$ $\operatorname{trd} L / R-\operatorname{trd}(L / N) /(R / M) ; z$ is a minimal prime ideal in $R^{*}$.

(2.11.2) (Cf. [9, Corollary 2.5].) If $R$ is quasi-unmixed, then $L$ is quasiunmixed.

Proof. (2.11.1) By (a) $\Longleftrightarrow(\mathrm{b}), c(L)=\{$ depth $w ; w$ is a minimal prime ideal in $\left.L^{*}\right\}$ and $c(R)=\left\{\right.$ depth $z ; z$ is a minimal prime ideal in $\left.R^{*}\right\}$. Therefore (2.11.1) follows from (2.5) and (2.4.3).

(2.11.2) follows from (2.11.1) and (2.4.3). Q.E.D.

It is known $[1,(10.13)]$ that, with $(L, N)$ and $(R, M)$ as in $(2.5)$, if $R$ is analytically irreducible, then $R$ is a subspace of $L$. (2.11.1) suggests that this might also be true if $R$ is unmixed (2.1.5). The author does not know if this is true.

3. A condition for $f: c(L) \rightarrow c(R)$ to be bijective. In this section we give a condition under which the converse of (2.5) holds; that is, in terms of the map $f$ of (2.6.1), for $f$ to be a bijection. Then we give some corollaries to the result.

To prove (3.1), we need the following result: If a local domain $L$ is a locality over a complete local domain, then $L$ is unmixed (2.1.5). This follows from [5, Proposition 4], as noted in the paragraph preceding (2.5) in [17].

(3.1) THEOREM. Let $(R, M)$ be a local domain and let $(L, N)$ be a locality over $R$ of the form: $L=R\left[X_{1}, \ldots, X_{k}, d_{1}, \ldots, d_{m}\right]_{P}(k \geqslant 0), d_{1}, \ldots$, $d_{m}$ in the quotient field of $R_{k}=R\left[X_{1}, \ldots, X_{k}\right]$, and $P \cap R=M$. Assume that $R$ is a subspace of $L$. Then $n \in c(L)$ if and only if $n-\operatorname{trd} L / R+$ $\operatorname{trd}(L / N) /(R / M)=n-k+\operatorname{depth} P \in c(R)$.

Proof. Let $L^{\circ}=A_{Q}$, where $A=R^{*}\left[X_{1}, \ldots, X_{k}, d_{1}, \ldots, d_{m}\right], R^{*}$ is the completion of $R$, and $Q=P A$ (so $Q$ is a prime ideal and $L$ is a dense sub. space of $L^{\circ} \quad\left[11\right.$, Lemma 3.2]). Since $R$ is a subspace of $L, R^{*}$ is a subring of the completion $L^{*}=L^{\circ *}$ of $L$ and of $L^{\circ}$; hence $Q$ contains all the prime divisors of zero in $A$ and $R^{*}$ is a subring of $L^{\circ}$ [11, Lemma 4.5(1)].

Now, $n \in c(L)$ if and only if (by (a) $\Longleftrightarrow(\mathrm{b})$ applied to $L$ ) there exists a depth $n$ minimal prime ideal in $L^{*}=L^{\circ *}$ if and only if (by the comment preceding this theorem) there exists a depth $n$ minimal prime ideal in $L^{\circ}$. Also, $z^{\prime}$ is a minimal prime ideal in $L^{\circ}$ if and only if (since $R^{*} \subseteq L^{\circ}$ and $Q$ contains all the prime divisors of zero in $A) z=\left(z^{\prime} \cap A\right) \cap R^{*}$ is a minimal prime ideal in $R^{*}$ and $z^{\prime}=z T\left(X_{1}, \ldots, X_{k}\right) \cap L^{\circ}$, where $T$ is the total quotient ring of $R^{*}$. 
Further, $L^{\circ} / z^{\prime}$ is a locality over the complete local domain $R^{*} / z$, and $R^{*} / z$ satisfies the altitude formula. Therefore altitude $L^{\circ} / z^{\prime}+\operatorname{trd}(A / Q) /\left(R^{*} / M^{*}\right)=$ altitude $R^{*} / z+k$; and depth $Q=$ altitude $A / Q=[6,(14.6)] \operatorname{trd}(A / Q) /\left(R^{*} / M^{*}\right)$, since $R^{*} / M^{*}$ is a field. Therefore depth $z^{\prime}=\operatorname{depth} z+k-\operatorname{depth} Q$. Hence, there exists a depth $n$ minimal prime ideal in $L^{\circ}$ if and only if there exists a depth $n-k+$ depth $Q$ minimal prime ideal in $R^{*}$; and this is true if and only if (by (a) $\Longleftrightarrow$ (b)) $n-k+$ depth $Q \in c(R)$. Finally, $R_{k}\left[d_{1}, \ldots, d_{m}\right] / P=A / Q$, so depth $P=\operatorname{depth} Q$. Q.E.D.

The condition that $R$ be a subspace of $L$ is stronger than is needed for the conclusion of (3.1), as will be seen in (6.7). However, the author knows of no other condition which always works.

If some $d_{i}$ in (3.1) is not in the quotient field of $R\left[X_{1}, \ldots, X_{k}\right]$, then $L$ need not be a dense subspace of $L^{\circ}$. For example, let $R$ be the regular local ring given in [6, (E3.2), p. 206], and let $d=d_{1}$ be the element $c$ in [6]. Then $d \in$ $R^{*}, d$ is integral over $R$, and $R[d]$ is a local domain which is not a dense subspace of $R^{*}[d]=R^{*}$.

It should be noted that, with $R$ and $L$ as in (3.1), if $R$ is a subspace of $L$, then the map $f$ of (2.6.1) is a bijection; hence (2.7.1) holds.

(3.2) REMARK. Let $(R, M)$ be a local domain. Then the following statements hold:

(3.2.1) (3.1) gives another proof of (a) $\Longleftrightarrow(d)$, since $R$ is a subspace of $\left(R_{k}\right)_{Q}$, by [11, Lemma $\left.4.5(1)\right]$.

(3.2.2) (Cf. $[17,(2.15)]$.) $c(R(X))=c(R)$, where $R(X)=R[X]_{M R[X]}$, since $R$ is a subspace of $R(X)$, by [11, Lemma $4.5(1)]$.

While on the subject of $R(X)$, it should be noted that $n \in c(R(X))$ does not imply that there exists a mcpil $n$ in $R(X)$ (in contrast to $n \in c(D)$ if and only if there exists a mcpil $n$ in $D$, where $\left.D=R[X]_{(M, X)}\right)$. For example, let $R$ be a catenary (2.1.2) local domain such that altitude $R>1$ and such that its integral closure has a height one maximal ideal (as in [6, Example 2, pp. 203-205]). Then $R(X)$ is catenary [12, Theorem 4.11] , altitude $R(X)>1$, and its integral closure has a height one maximal ideal.

(3.3) REMARK. Let $(R, M)$ and $(L, N)$ be as in (3.1), and assume that $R$ is a subspace of $L$. Then the following statements hold:

(3.3.1) $R$ is quasi-unmixed if and only if $L$ is quasi-unmixed.

(3.3.2) $R$ satisfies the s.c.c. if and only if $L$ satisfies the s.c.c.

Proof. (3.3.1) follows from (3.1) and (2.4.3); and (3.3.2) follows from (3.1) and (2.4.2). Q.E.D.

To prove the next corollary to (3.1), we need some information on Rees rings.

(3.4) Definition. Let $I=\left(d_{1}, \ldots, d_{m}\right) R$ be an ideal in a local domain 
$(R, M)$. Then the Rees ring $R=R(R, I)$ of $R$ with respect to $I$ is defined to be the subring $R=R\left[t d_{1}, \ldots, t d_{m}, u\right]$ of $R[t, u]$, where $t$ is an indeterminate and $u=1 / t$. The ideal $M=\left(t d_{1}, \ldots, t d_{m}, M, u\right) R$ is called the maximal homogeneous (irrelevant) ideal in $R$.

We summarize in the following remark the known results on Rees rings which will be needed in the remainder of this paper.

(3.5) Remark. Let the notation be as in (3.4).

(3.5.1) $R$ is a graded Noetherian domain, and the elements in $R$ are finite sums $\Sigma_{-k}^{n} c_{j} t^{j}$, where $c_{j} \in I^{j}$ (with the convention that $I^{j}=R$, if $j \leqslant 0$ ). Moreover, $u^{j} R \cap R=I^{j}(j \geqslant 0)$.

(3.5.2) $M$ is a maximal ideal in $R$ and every homogeneous ideal in $R$ is contained in $M$ [20, Theorem 3.1 (step (ii))].

(3.5.3) If $H$ is a maximal relevant ideal in $R$, then $H$ is prime and $(M, u) R$ $\subseteq H \subset M$, and depth $H=1$ [20, Theorem 2.1]. (A homogeneous ideal $H$ in $R$ is said to be irrelevant in case $H$ contains all homogeneous elements of sufficiently large degree; otherwise $H$ is said to be relevant. $H$ is a maximal relevant ideal in case $H$ is maximal with respect to being relevant.)

(3.5.4) The form ring $F(R, I)$ of $R$ with respect to $I$ is isomorphic to $R / u R$ $[19$, Theorem 2.1].

(3.6) Corollary. Let I be an ideal in a local domain $R$, let $R=R(R, I)$ be the Rees ring of $R$ with respect to $I$, and let $L=R_{M}$, where $M$ is the maximal irrelevant ideal in $R$. Then $n \in c(R)$ if and only if $n+1 \in c(L)$.

Proof. Let $R^{*}$ be the completion of $R$, let $R^{\circ}=R\left(R^{*}, I R^{*}\right)$, and let $M^{\circ}$ be the maximal irrelevant ideal in $R^{\circ}$. Then $M^{\circ}$ contains all prime divisors of zero in $R^{\circ}$ (since $M^{\circ}$ contains all homogeneous ideals in $R^{\circ}$ and the prime divisors of zero are homogeneous). Therefore, since $R$ is contained in the quotient field of $R[t], R$ is a subspace of $L$ [11, Lemma 4.5(1)]. Therefore the conclusion follows from (3.1). Q.E.D.

We close this section of the paper with one further result on Rees rings and $c(R)$. More will be said about (3.7) in the next section of the paper.

(3.7) Proposition. Let $(R, M)$ be a local domain. Then there exists an $M$-primary ideal $B$ in $R$ such that, with $R=R(R, B)$ and $M$ the maximal irrelevant homogeneous ideal in $R$, the following statements are equivalent:

(3.7.1) $n \in c(R)$.

(3.7.2) $n+1 \in c\left(R_{M}\right)$.

(3.7.3) There exists a maximal relevant ideal $N$ in $R$ such that height $N=n$.

(3.7.4) There exists a minimal prime divisor $p$ of $u R$ such that depth $p=$ height $M / p=n$. 
(3.7.5) There exists a minimal prime ideal $z$ in the form ring $\dot{F}=F(R, B)$ such that depth $z=n$.

Proof. We note, to begin with, that if $B=\left(b_{1}, \ldots, b_{m}\right) R$ is an $M$ primary ideal and $p$ is a minimal prime divisor of $u R(R, B)$, then $B=u R \cap R$ $\subseteq p \cap R$, so depth $p=$ height $M / p$ (since $u R$ is homogeneous implies $p \subseteq M$, and since depth $p=$ altitude $R / p$ and $R / p$ is a homomorphic image of $(R / M)\left[X_{1}, \ldots, X_{m}\right]=$ (say) $D$, and $D$ satisfies the s.c.c.).

Now, by [11, Proposition 3.9], there exists an $M$-primary ideal $B$ in $R$ such that the following sets are equal: \{depth $z ; z$ is a minimal prime ideal in the completion of $R$ \}; \{depth $p ; p$ is a minimal prime divisor of $u R(R, B)$ \}; and, \{ height $N ; N$ is a maximal relevant ideal in $R(R, B)$ \}. Therefore, for this $B$, since depth $p=$ height $M / p$ (by the preceding paragraph), (3.7.3) and (3.7.4) are equivalent, and each is equivalent to (3.7.1), by (a) $\Longleftrightarrow(\mathrm{b})$. Also, (3.7.1) and (3.7.2) are equivalent, by (3.6). Finally, (3.7.4) and (3.7.5) are equivalent, since $F \cong R / u R$ (3.5.4). Q.E.D.

4. The class of local domains $(R, M)$ with $c(R)=W(0, M)$. Let $C$ be the class of local domains $(R, M)$ such that $\{m$; there exists an integral extension domain of $R$ which has a mcpil $m\}=\{n$; there exists a mcpil $n$ in $R\}$; that is, such that $c(R)=W(0, M)($ see (2.3)). In this section we will give some information on $C$.

Our first result on $C$ gives some knowledge of the rings which are in $C$.

(4.1) Proposition. The following statements hold:

(4.1.1) If $R$ satisfies the s.c.c., then $R \in C$.

(4.1.2) For all local domains $R, R[X]_{Q} \in C$, for all maximal ideals $Q$ in $R[X]$ such that $Q \cap R=M$.

(4.1.3) If $R$ is Henselian, then $R \in C$.

(4.1.4) For all local domains $R$, there exist $M$-primary ideals $B$ in $R$ such that $R_{M} \in C$, where $R=R(R, B)$.

(4.1.5) If $R \subseteq S$ are local domains such that $S$ is integral over $R$, then $R \in$ $C$ if and only if $S \in C$.

(4.1.6) $R \in C$ if and only if $R(X) \in C$.

(4.1.7) If $R \in C$ and $p$ is a prime ideal in $R$, then it is not necessarily true that $R / p$ or $R_{p}$ is in $C$.

Proof. (4.1.1) If $R$ satisfies the s.c.c., then $c(R)$ is a one-point set (2.4.2); hence $c(R)=W(0, M)$, and so $R \in C$.

(4.1.2) follows from (d) $\Longleftrightarrow(\mathrm{e})$, since $M R[X] \subset Q$, if $Q$ is maximal and $Q \cap R=M$.

(4.1.3) is given in $[15,(3.2)]$. 
(4.1.4) follows from (3.7.2) $\Longleftrightarrow$ (3.7.4).

(4.1.5) is given in $[15,(3.14)]$.

(4.1.6) $c(R)=c(R(X))$ (3.2.2). Also, $W(0, M)=W(0, M R(X))[2$, Theorem 6]. Therefore, $R \in C$ if and only if $R(X) \in C$.

(4.1.7) Let $(R, M)$ be a local domain which is not in $C$. (For example, let $R$ be as in [6, Example 2, pp. 203-205] in the case $m=0$.) Let $D=R[X]_{(M, X)}$. Then $D \in C$ (4.1.2), but $R=D / X D \notin C$ and $R(X)=D_{M D} \notin C$ (by (4.1.6)). Q.E.D.

Our next result gives some properties of each ring in $C$.

(4.2) Proposition. The following statements hold for $(R, M) \in C$ :

(4.2.1) For all prime ideals $Q$ in $R[X]$ such that $Q \cap R=M, R[X]_{Q} \in C$.

(4.2.2) There exists a depth $n$ minimal prime ideal in the completion of $R$ if and only if there exists a prime ideal $p$ in $R$ such that height $p=n-1$ and depth $p=1$.

(4.2.3) If $R$ is catenary, then $R$ satisfies the s.c.c.

(4.2.4) If $(L, N)$ is a locality over $R$ such that $N \cap R=M$, and if $n \in$ $c(L)$, then $n-\operatorname{trd} L / R+\operatorname{trd}(L / N) /(R / M) \in W(0, M)$.

(4.2.5) If $(L, N)$ is as in (3.1) and $R$ is a subspace of $L$, then $n \in c(L)$ if and only if $n-\operatorname{trd} L / R+\operatorname{trd}(L / N) /(R / M) \in W(0, M)$.

Proof. (4.2.1) If $M R[X] \subset Q$, then $R[X]_{Q} \in C$, by (4.1.2). If $M R[X]$ $=Q$, then $R[X]_{Q}=R(X) \in C$, by (4.1.6).

(4.2.2) It is known that if there exists a mcpil $n$ in a local domain $R$, then there exists a prime ideal $p$ in $R$ such that height $p=n-1$ and depth $p=1$ [2, Theorem 5]. The conclusion follows from this and (a) $\Longleftrightarrow(\mathrm{b})$.

(4.2.3) If $R$ is catenary, then $c(R)=W(0, M)=\{$ altitude $R\}$; hence $R$ satisfies the s.c.c. (2.4.2).

(4.2.4) is clear by (2.5).

(4.2.5) is clear by (3.1). Q.E.D.

An easy proof of the following known result can now be given.

(4.3) Corollary (CF. [11, TheOREM 2.21]). A catenary Henselian local domain satisfies the s.c.c.

Proof. This follows from (4.1.3) and (4.2.3). Q.E.D.

(4.4) Remark. It follows from (4.3) that a local Henselian domain $R_{1}$ of altitude two satisfies the s.c.c. Also, if altitude $R=3$ and $R$ is $H_{1}$ (that is, height one prime ideals have depth two), then $R$ satisfies the s.c.c. (since $R$ is catenary). In particular, if $R$ is a local Henselian UFD of altitude three, then $R$ satisfies the s.c.c. (since height one prime ideals are principal implies that $R$ is $H_{1}$ ). The author does not know if all local UFD's of altitude three satisfy the s.c.c. 
Another proof that an altitude three Henselian local domain which is $H_{1}$ satisfies the s.c.c. (equivalently, is quasi-unmixed [10, Theorem 3.1]) is given in (5.5.2) below.

The author suspects that $C$ contains most local domains. (See (4.10.3) for one reason.) In any case, there are some interesting problems in this area. We mention three of these in (4.5)-(4.7). To explain the first problem, let $R$ be a local domain. Then $c(R)$ is a finite set, so, by the going-up theorem and [17, (2.2)], there exists a finite integral extension domain $S$ of $R$ such that $n \in c(R)$ if and only if there exists a mcpil $n$ in $S$. Therefore, if $S$ is local, then $S \in C$, hence $R \in C$ (4.1.5). ( $S \in C$, since, if $A \subseteq B$ are integral domains such that $B$ is integral over $A$, then $c(B) \subseteq c(A)$.) This leads to the following question which the author has not been able to answer:

(4.5) Question. With the preceding notation, if $N$ is a maximal ideal in $S$, is $S_{N} \in C$ ?

More generally, we ask:

(4.6) Question. If $R \in C$ and $N$ is a maximal ideal in a finite integral extension domain $S$ of $R$, is $S_{N} \in C$ ?

(4.7) Question. If $R \in C$, if $L$ is as in (3.1), and if $R$ is a subspace of $L$, is $L \in C ?$

A partial answer to (4.6) is given in (4.8.2).

(4.8) REMARK. Let $(R, M)$ be a local domain.

(4.8.1) If $R$ has the property that, for each integral extension domain $S$ of $R$, each maximal chain of prime ideals in $S$ contracts in $R$ to a maximal chain of prime ideals, then $R \in C$.

(4.8.2) If $R \in C$ and the integral closure $R^{\prime}$ of $R$ is quasi-local, then, for each integral extension domain $S$ of $R$, and for each maximal ideal $N$ in $S, S_{N} \in$ $C$ and $c\left(S_{N}\right)=c(R)$.

Proof. (4.8.1) is clear.

(4.8.2) Let $S$ be an integral extension domain of $R$ and let $N$ be a maximal ideal in $S$. Let $S^{\prime}$ be a Galois (not necessarily separable) extension domain of $R$ which contains $S$, and let (0) $\subset p_{1} \subset \cdots \subset p_{n}=M$ be a mcpil $n$ in $R$. Then there exists a covering chain of prime ideals (0) $\subset P_{1} \subset \cdots \subset P_{n}=N^{\prime}$ in $S^{\prime}$. Let $N^{\prime \prime}$ be a maximal ideal in $S^{\prime}$ such that $N^{\prime \prime} \cap S=N$. Then, since $N^{\prime}$ and $N^{\prime \prime}$ are conjugate [6, (10.12)] (since $R^{\prime}$ is quasi-local), there exists a mcpil $n$ in $S^{\prime}$, say (0) $\subset Q_{1} \subset \cdots \subset Q_{n}=N^{\prime \prime}$ such that $Q_{i} \cap R=p_{i}(i=1, \ldots, n)$. Therefore, with $q_{i}=Q_{i} \cap S,(0) \subset q_{1} \subset \cdots \subset q_{n}=N$ is a mcpil $n$ contained in $N$. (The chain is maximal, since $q_{i} \cap R=p_{i}$ and (0) $\subset p_{1} \subset \cdots \subset p_{n}=M$ is maximal.) Therefore $c(R) \subseteq W\left(0, N S_{N}\right)$ and $W\left(0, N S_{N}\right) \subseteq c\left(S_{N}\right) \subseteq c(R)$, by (2.9). Hence $S_{N} \in C$ and $c\left(S_{N}\right)=c(R)$. Q.E.D.

One further problem in this area (related to (4.8.1)) will now be mentioned. 
(4.9) Question. If $R \subset S$ are local domains such that $S$ is integral over $R$, then does every maximal chain of prime ideals in $S$ contract in $R$ to a maximal chain of prime ideals?

Using induction on altitude $R$, it suffices to prove that if $P$ is a height one prime ideal in $S$, then height $P \cap R=1$.

(4.10.3) contains some comments relating the upper conjecture to the class C. For further comments on this conjecture, see [3, Propositions 3.3 and 3.7] and $[17,(2.21)$ and $(2.22)]$.

(4.10) REMARK. The following statements hold for a local domain $(R, M)$ :

(4.10.1) If $R \in C, n \in c(R)$, and $n>1$, then there exist infinitely many prime ideals $p$ in $R$ such that height $p=n-1$ and depth $p=1$.

(4.10.2) If $R \in C, n \in c(R)$, and $n=1$, then altitude $R=1$.

(4.10.3) The following statements are equivalent: (i) The upper conjecture holds. (That is, $\{n+1 ; n \in W(0, M)\} \subseteq W\left(1,\left(M, X_{1}\right)\right) \subseteq\{n+1 ; n \in W(0, M)\}$ $\cup\{2\}$.) (ii) $W(0, M) \subseteq c(R) \subseteq W(0, M) \cup\{1\}$. (iii) If $R \notin C$, then $W(0, M)$ $\subset c(R)=W(0, M) \cup\{1\}$. (iv) If $R \notin C$, then altitude $R>1$ and there exists height one maximal ideal in the integral closure $R^{\prime}$ of $R$.

Proof. (4.10.1) It is known that if there exists a prime ideal $p$ in a local domain, such that height $p=h>0$ and depth $p=d>0$, then there exist infinitely many such prime ideals $[12,(2.2 .1)]$. Therefore the conclusion follows from (4.2.2) and (a) $\Longleftrightarrow(b)$.

(4.10.2) follows from (4.2.2) and (a) $\Longleftrightarrow(b)$.

(4.10.3) Assume that (i) holds and let $R$ be a local domain. Then clearly $W(0, M) \subseteq c(R)$. Also, if $n \in c(R)$, then $n+1 \in W\left(1,\left(M, X_{1}\right)\right)$ (by (2.4.4)), so, by (i), $n \in W(0, M)$ or $n=1$; hence $c(R) \subseteq W(0, M) \cup\{1\}$. Thus (i) $\Longrightarrow$ (ii).

Assume that (ii) holds and let $(R, M)$ be a local domain such that $R \notin C$. Then $W(0, M) \subset c(R)$, so $c(R)=W(0, M) \cup\{1\}$, by (ii); hence (ii) $\Rightarrow$ (iii).

Next, assume that (iii) holds and $R \notin C$. Then clearly altitude $R>1$. Also, there exists an integral extension domain of $R$ which has a height one maximal ideal (by (iii)), so [12, Lemma 2.9] says there exists a height one maximal ideal in $R^{\prime}$. Thus (iii) $\Rightarrow$ (iv).

To show that (iv) $\Rightarrow$ (iii), let $(R, M)$ be a local domain $\notin C$, so $W(0, M) \subset$ $c(R)$. Then, by (iv), $1 \in c(R), \notin W(0, M)$. Also, by [15, (4.7)], there exists a local domain $(S, N)$ such that $W(0, M)=W(0, N)$ and $c(S) \subset c(R)=c(S) \cup$ $\{1\}$. Then, by (iv), $S \in C$ (since $1 \notin c(S)$ ), so $c(R)=c(S) \cup\{1\}=W(0, N) \cup$ $\{1\}=W(0, M) \cup\{1\}$.

Finally, assume that (iii) holds and let $R$ be a local domain. It may clearly be assumed that $\{n+1 ; n \in W(0, M)\} \subset W\left(1,\left(M, X_{1}\right)\right)$; that is, by (2.4.4), that $W(0, M) \subset c(R)$, so $R \notin C$. Therefore, by (iii) and $(2.4 .4), W\left(1,\left(M, X_{1}\right)\right)=$ 
$\{n+1 ; n \in W(0, M)\} \cup\{2\}$, hence (iii) $\Rightarrow$ (i). Q.E.D.

We close this section with a comment showing the importance of knowing more properties of the rings in $C$. Namely, let $(R, M), B$, and $R_{M}$ be as in (3.7). Then $R_{M} \in C$, by (3.7.2) $\Longleftrightarrow(3.7 .4)$. Also, by (3.7.3) $\Longleftrightarrow$ (3.7.4) (and (3.5.3)), there exists a height $n$ depth one prime ideal in $R_{M}$ if and only if there exists a height one depth $n$ prime ideal in $R_{M}$. If every $R \in C$ (or, even, if every $\left.R[X]_{(M, X)} \in C\right)$ had this property, then it can be shown that the $H$-conjecture (that is, an $H_{1}$-local domain (see (5.3)) is catenary) holds. (See [14, (4.5)], where it is shown that the $H$-conjecture implies the catenary chain conjecture (that is, the integral closure of a catenary local domain satisfies the c.c. (2.1.4)).) (In this regard, there are two conditions, either of which implies that, with $(R, M)$ a local domain, if $n \in c(R)$, then there exists a height one depth $n-1$ prime ideal in $R$. Namely, there exists $b \in M$ such that either: (a) every prime divisor $p$ of $b R$ is such that height $p=1$ and $R / p$ is unmixed; or, (b) for all minimal prime ideals $z$ in the completion $R^{*}$ of $R$, height $(z, b) R^{*}=1$ and, for all minimal prime divisors $p$ of $b R, R / p$ is quasi-unmixed. Does every $R[X]_{(M, X)} \in C$ satisfy one of these conditions?)

5. Two more equivalences of (a)-(f). In this section we give, in (5.2), two new equivalences of the six statements (a)-(f) in the introduction, and we extend the equivalence of (b) and (f) to the case $R$ contains nonzero divisors of zero. Then some corollaries of (5.2) are given which relate the results to $H_{i}$-rings ((5.4) and (5.6)), and this section is closed by characterizing all local domains $R$ such that card $c(R) \leqslant 2 \quad((5.9)$ and $(5.10))$.

To prove (5.2), we need a result which was essentially proved in [10, Proposition 2.16]. Unfortunately, the hypothesis in [10, Proposition 2.16] is not general enough for our present need, so we prove it anew in (5.1).

(5.1) Lemma (CF. [10, Proposition 2.16]). Let $\left(R^{*}, M^{*}\right)$ be the completion of a local ring $R$, and let $z$ be a minimal prime ideal in $R^{*}$ such that depth $z$ $=n>1$. Then, for each $i=1, \ldots, n-1$, there exist infinitely many prime ideals $q$ in $R$ such that height $q=i$ and $q R^{*}$ has a minimal prime divisor $P$ such that $z \subset P$, height $P=$ height $P / z=i$, and depth $P=n-i$.

Proof. Let $b \in M, \notin z \cap R$. Then there exists a prime ideal $P$ in $R^{*}$ such that $(z, b) R^{*} \subseteq P$ and height $P / z=1$ (by the principal ideal theorem in $\left.R^{*} / z\right)$. Therefore, if $S=\left\{P \in \operatorname{Spec} R^{*} ; z \subset P\right.$ and height $\left.P / z^{*}=1\right\}$, then $M=$ $\bigcup\{P \cap R ; P \in S\}$. Also, $M^{*} \notin S$, since depth $z>1$; hence $S$ is an infinite set. Further, only finitely many $P \in S$ are such that height $P>$ height $z+1=1$ $[2$, Theorem 1], so there exist infinitely many $P \in S$ such that height $P=$ height $P / z=1$ and $z \cap R \subset P \cap R=$ (say) $q$. Therefore height $q \geqslant 1$, so $1=$ 
height $P \geqslant$ height $q R^{*}=[6,(22.9)]$ height $q \geqslant 1$; hence height $q=1$ and $P$ is a minimal prime divisor of $q R^{*}$. Also, depth $P=\operatorname{depth} P / z=n-1$ (since $R^{*} / z$ is catenary). Then $R^{*} / q R^{*} \cong(R / q)^{*}$, the completion of $R / q$, and there exists a depth $n-1$ minimal prime ideal in $R^{*} / q R^{*}$.

If $n-1>1$, then fix one of the $P$ and $q=P \cap R$ and repeat the above for $R / q$. Then it follows that there exist infinitely many prime ideals $p$ in $R$ such that $q \subset p$, height $p / q=1$, and $p R^{*}$ has a minimal prime divisor $p^{*}$ such that $P \subset p^{*}$ and height $p^{*} / P=1$ (so height $p^{*} / z=2$, since $R^{*} / z$ is catenary). Then only finitely many of the $p^{*}$ are such that height $p^{*}>$ height $P+1[2$, Theorem 1], so infinitely many of the $p^{*}$ are such that $2=$ height $p^{*} / z=$ height $p^{*}=$ height $p$; and depth $p^{*}=n-2$. Therefore, the conclusion follows from a finite number of repetitions. Q.E.D.

The following theorem is the main result of this section. It gives two additional equivalences of (a)-(f) of the introduction in the local domain case, and it extends the equivalence of $(b)$ and $(f)$ to local rings with zero divisors.

(5.2) THEOREM. Let $n$ be a positive integer. Then the following statements are equivalent for a local ring $(R, M)$ :

(5.2.1) There exists a minimal prime ideal $z$ in the completion $R^{*}$ of $R$ such that depth $z=n$.

(5.2.2) There exists a prime ideal $P$ in $D=R[X]_{(M, X)}$ such that height $P$ $=n$ and depth $P=1$.

(5.2.3) There exists a prime ideal $q$ in the Henselization $R^{H}$ of $R$ such that height $q=n-1$ and depth $q=1$.

(5.2.4) There exists a prime ideal $p$ in $R$ such that height $p=n-1$ and such that the integral closure of $R / p$ has a height one maximal ideal.

Proof. (5.2.1) $\Rightarrow(5.2 .2)$. Assume that (5.2.1) holds, let $z$ be a depth $n$ minimal prime ideal in $R^{*}$, and let $w=z \cap R$, so $z$ is a minimal prime divisor of $w R^{*}$. Then $R / w$ is a local domain, $R^{*} / w R^{*} \cong(R / w)^{*}$, the completion of $R / w$, and $D / w D \cong(R / w)[X]_{(M / w, X)}$, so, by (b) $\Longleftrightarrow(f)$, there exists a mcpil $n+1$ in $D$ with $w D$ as the smallest term. Then, by [4, Lemma 1], there exists a mcpil $n+1$ in $D$, say $w D=p_{0} \subset \cdots \subset p_{n+1}=(M, X) D$ such that height $p_{i}=i(i<n+1)$. Therefore height $p_{n}=n$ and depth $p_{n}=1$; hence (5.2.2) holds.

(5.2.2) $\Rightarrow$ (5.2.1) Assume that (5.2.2) holds, let $p$ be a height $n$ prime ideal in $D$ such that depth $p=1$, and let $w$ be a minimal prime ideal in $R$ such that $w \subset p$ and height $p / w=n$. Then, clearly, there exists a mcpil $n+1$ in $D / w D$, so there exists a depth $n$ minimal prime ideal in the completion $(R / w)^{*}$ $\cong R^{*} / w R^{*}$ of $R / w$, by (b) $\Longleftrightarrow$ (f), and so there exists a depth $n$ minimal prime ideal in $R^{*}$, as desired. 
(5.2.1) $\Rightarrow(5.2 .4)$ Let $z$ be a depth $n$ minimal prime ideal in $R^{*}$, and let $w$ $=z \cap R$, so the completion $(R / w)^{*}$ of $R / w$ is isomorphic to $R^{*} / w R^{*}$. Now, if $n=1$, then there exists a height one maximal ideal in the integral closure of $R / w$ [10, Proposition 3.5] and height $w=0$. Therefore it may be assumed that $n>$ 1. Then, by (5.1), there exists a prime ideal $p$ in $R$ such that height $p=n-1$ and $p R^{*}$ has a minimal prime divisor $p^{*}$ such that $z \subset p^{*}$ and height $p^{*}=$ height $p^{*} / z=n-1$. Then depth $p^{*}=\operatorname{depth} p^{*} / z=1$ (since depth $z=n$ and $R^{*} / z$ is catenary); hence the completeion $(R / p)^{*} \cong R^{*} / p R^{*}$ of $R / p$ has a depth one minimal prime ideal. Therefore, there exists a height one maximal ideal in the integral closure of $R / p$ [10, Proposition 3.5] , and so (5.2.4) holds.

(5.2.4) $\Rightarrow$ (5.2.1) Assume that (5.2.4) holds. Then there exists a depth one minimal prime ideal in $R^{*} / p R^{*}\left[10\right.$, Proposition 3.5]; hence $p R^{*}$ has a depth one minimal prime divisor $p^{*}$, and necessarily height $p^{*}=$ height $p=n-1$. Therefore, if $z$ is a minimal prime ideal in $R^{*}$ such that $z \subseteq p^{*}$ and height $p^{*} / z=n$ - 1 , then depth $z=n$ (since $R^{*} / z$ is catenary). Therefore (5.2.1) holds.

(5.2.1) $\Longleftrightarrow(5.2 .3) R^{H}$ is a local ring and $R^{*}$ is the completion of $R^{H}$ $[6,(43.10)]$, so, by $(5.2 .1) \Longleftrightarrow(5.2 .4)$, there exists a depth $n$ minimal prime ideal in $R^{*}$ if and only if there exists a prime ideal $q$ in $R^{H}$ such that height $q=$ $n-1$ and the integral closure $I$ of $R^{H} / q$ has a height one maximal ideal. Now $R^{H} / q$ is a Henselian local domain, so there exists a height one maximal ideal in $I$ if and only if depth $q=$ altitude $R^{H} / q=1$. Q.E.D.

(5.3) Definition. A ring $A$ is said to be an $H_{i}$-ring (or, $A$ is said to be $H_{i}$ ) in case, for each height $i$ prime ideal $p$ in $A$, depth $p=$ altitude $A-i$. (That is, height $p+\operatorname{depth} p=$ altitude $A$.)

Numerous properties of $H_{i}$-local domains are given in [7] and [8], and some additional properties of such rings are given in [14] and [18]. There are a number of reasons why such rings are of importance. Here, we mention only two reasons: such rings led to the $H$-conjecture (that is, an $H_{1}$-local domain is catenary) which, like the upper conjecture, is implied by the depth conjecture and implies the catenary chain conjecture (see [13, §3] and [16]); and, if every Henselian local domain is an $H_{1}$-ring, then the chain conjecture holds $[14,(2.4)]$.

The following corollary relates (5.2) to certain local rings being $H_{n}$-rings.

(5.4) CoROllaRY. With the notation of (5.2) and with $a=$ altitude $R$, the following statements hold:

(5.4.1) If $R$ is $H_{n}(n<a)$, then either $R$ is $H_{n-1}$ or there exists a depth $n$ minimal prime ideal in $R^{*}$.

(5.4.2) If $D$ is $H_{n+1}(n<a)$, then either $D$ is $H_{n}$ or there exists a depth $n$ minimal prime ideal in $R^{*}$. 
(5.4.3) If $R^{H}$ is $H_{n}(n<a-1)$, then there does not exist a depth $n+1$ ninimal prime ideal in $R^{*}$. Moreover, either $R^{H}$ is $H_{n-1}$ or there exists a depth $n$ minimal prime ideal in $R^{*}$.

(5.4.4) If $D$ is $H_{n}(n<a)$, then there does not exist a depth $n$ minimal prime ideal in $R^{*}$.

(5.4.5) If $R$ is Henselian and $D$ is $H_{n}(n<a-1)$, then there does not exist a minimal prime ideal $z$ in $R^{*}$ such that depth $z \in\{n, n+1\}$.

Proof. (5.4.1) Assume that $R$ is not $H_{n-1}$. Then there exists a prime ideal $p$ in $R$ such that height $p=n-1$ and depth $p<a-n+1$. Suppose depth $p>1$. Then $p$ is contained in infinitely many prime ideals; hence there exists a prime ideal $P$ in $R$ such that $p \subset P$ and height $P=n$ [2, Theorem 1]. Hence, since $R$ is $H_{n}$, depth $P=a-n$, and so depth $p \geqslant a-n+1$; contradiction. Therefore depth $p=1$; hence there exists a depth $n$ minimal prime ideal in $R^{*}$ (by (5.2.1) $\Longleftrightarrow(5.2 .4)$ ).

(5.4.2) The proof is similar to that of (5.4.1), using (5.2.1) $\Longleftrightarrow(5.2 .2)$.

(5.4.3) The first statement is clear by $(5.2 .1) \Longleftrightarrow(5.2 .3)$. The second statement follows from (5.4.1) applied to $R^{H}$.

(5.4.4) is clear by (5.2.1) $\Longleftrightarrow(5.2 .2)$.

(5.4.5) By (5.5.1) below, if $D$ is $H_{n}$, then $R$ is $H_{n}$ and $H_{n-1}$, so the conclusion follows from (5.4.3). Q.E.D.

(5.5) REMARK. (5.5.1) It is known [18, (3.7)] that $D$ is $H_{n+1}$ if and only if $R$ is a $C_{n}$-ring (that is, $R$ is $H_{n}, H_{n+1}$, and, for each height $n$ prime ideal $p$ in $R$, all maximal ideals in the integral closure of $R / p$ have the same height (= altitude $R / p=$ depth $p$ )). Therefore, another way of stating (5.4.2) is: If $R$ is a $C_{n}$-ring, then either $R$ is a $C_{n-1}$-ring (that is (since $R$ is $H_{n}$ ) $R$ is $H_{n-1}$ and, for each height $n-1$ prime ideal $q$ in $R$, all maximal ideals in the integral closure of $R / q$ have the same height), or there exists a height $n-1$ prime ideal $q$ in $R$ such that there exists a height one maximal ideal in the integral closure of $R / q$ (by (5.4.2) and (5.2.1) $\Longleftrightarrow(5.2 .4)$ ).

(5.5.2) If $R$ is a Henselian local domain, altitude $R=3$, and $R$ is $H_{1}$, then $R$ is quasi-unmixed. (This follows easily from (5.4.3) and (5.2.1) $\Longleftrightarrow(5.2 .3)$.) (In this regard, see (4.4).)

The following corollary considers the case when there are no minimal prime ideals $z$ in the completion of $R$ with depth $z \in\{n, n+1, \ldots, a-1\}$.

(5.6) COROLLARY. With the notation of (5.2), the following statements are equivalent for $n<a=$ altitude $R$ :

(5.6.1) If $z$ is a minimal prime ideal in $R^{*}$, then either depth $z=a$ or depth $z<n$. 
(5.6.2) $D$ is $H_{n}, H_{n+1}, \ldots, H_{a+1}$.

(5.6.3) $R^{H}$ is $H_{n-1}, H_{n}, \ldots, H_{a}$.

(5.6.4) $R$ is $H_{n-1}, H_{n}, \ldots, H_{a}$ and, for each prime ideal $p$ in $R$ such that height $p \geqslant n-1$, all maximal ideals in the integral closure of $R / p$ have the same height.

(5.6.5) For each prime ideal $P$ in $D$ such that height $P \geqslant n$, depth $P=$ $(a+1)-$ height $P$ and $D / P$ is quasi-unmixed.

(5.6.6) For each prime ideal $q$ in $R^{H}$ such that height $q \geqslant n-1$, depth $q$ $=a$-height $q$ and $R^{H} / q$ is quasi-unmixed.

(5.6.7) For each prime ideal $p$ in $R$ such that height $p \geqslant n-1$, depth $p$ $=a-$ height $p$ and $R / p$ is quasi-unmixed.

Proof. (5.6.1) $\Rightarrow(5.6 .2)$, by (5.4.2), since altitude $D=a+1$ implies $D$ is $H_{a}, H_{a+1}$.

(5.6.2) and (5.6.4) are equivalent, as noted in (5.5.1).

(5.6.4) $\Rightarrow(5.6 .7)$ Assume that (5.6.4) holds and let $p$ be a prime ideal in $R$ such that height $p \geqslant n-1$. Then depth $p=a$ - height $p=$ (say) $d$ and $R / p$ is $H_{1}, \ldots, H_{d}[18,(2.4)]$; hence $R / p$ is catenary [13, Theorem 2.2]. Therefore, since, for each prime ideal $q$ in $R / p$ such that depth $q>1$, there are no height one maximal ideals in the integral closure of $(R / p) / q, R / p$ is quasi-unmixed (by [11, Theorem 2.19] and [10, Theorem 3.1]). Therefore (5.6.7) holds.

(5.6.7) $\Rightarrow(5.6 .5)$ Assume that (5.6.7) holds, and let $P$ be a prime ideal in $D$ such that height $P \geqslant n$. Then depth $P=(a+1)-$ height $P$, by (5.5.1). Also, height $P \cap R \geqslant n-1$, so $R /(P \cap R)$ is quasi-unmixed; hence $D / P$ is quasi-unmixed $[10$, Corollary 3.7], and so (5.6.5) holds.

(5.6.5) $\Rightarrow$ (5.6.1) Assume that (5.6.5) holds and let $z^{*}$ be a minimal prime ideal in the completion of $D$. Assume that depth $z^{*}<a+1$, and suppose that $d=$ depth $z^{*}>n$. Then, as in the proof of (5.2.1) $\Rightarrow(5.2 .4)$, there exists a height $d-1$ prime ideal $P$ in $D$ such that there is a height one maximal ideal in the integral closure of $D / P$. Now $a>d-1 \geqslant n$, so either $D / P$ is not quasi-unmixed or depth $P=1$; and this contradicts (5.6.5). Therefore depth $z^{*} \leqslant n$. Now let $D^{\prime}=R^{*}[X]_{\left(M^{*}, X\right)}$, so $D$ is a dense subspace of $D^{\prime}[11$, Lemma 3.2]. Therefore, since the minimal prime ideals in $D^{\prime}$ are the ideals $z D^{\prime}$ with $z$ a minimal prime ideal in $R^{*}$, since depth $z D^{\prime}=$ depth $z+1$, and since $D^{\prime} / z D^{\prime}$ is unmixed (see the comment preceding (3.1)), it follows that if depth $z<a$, then depth $z \leqslant n-1$. Therefore (5.6.1) holds.

(5.6.1) $\Rightarrow(5.6 .3)$, by (5.4.1) applied to $R=R^{H}$, since $R^{H}$ is $H_{a}$ and $H_{a-1}$. (5.6.3) implies (5.6.6), as in the proof that (5.6.4) $\Rightarrow(5.6 .7)$.

Finally, if (5.6.6) holds and $z$ is a minimal prime ideal in $R^{*}=\left(R^{H}\right)^{*}$ such that depth $z=d \geqslant n$, then, by (5.1), there exists a height $d-1$ prime ideal 
$p$ in $R^{H}$ such that depth $p=1$ (since $R^{H} / p$ is Henselian), so $d=a(5.6 .6)$, hence (5.6.1) holds. Q.E.D.

It follows from (5.6) that if $n-1$ is the largest integer $i<a$ such that there exists a depth $i$ minimal prime ideal in $R^{*}$, then, for each prime ideal $p$ in $R$ such that height $p \geqslant n-1$, depth $p=a-$ height $p$ and $R / p$ is quasi-unmixed. In particular, we have the following remark.

(5.7) REMARK. The following statements are equivalent: (a) There does not exist a depth $a-1$ minimal prime ideal in $R^{*}$. (b) $D$ is $H_{a-1}$. (c) $R^{H}$ is $H_{a-2}$. (d) For each height $a-2$ prime ideal $p$ in $R, R / p$ is quasi-unmixed and. altitude $R / p=2$.

Proof. Clear by (5.6.1) $\Longleftrightarrow(5.6 .2) \Longleftrightarrow(5.6 .3) \Longleftrightarrow$ (5.6.7). Q.E.D.

We next note that two known results follow readily from (5.6).

(5.8) REMARK. Let $(R, M)$ be a local ring, and let $a=$ altitude $R$. Then the following statements hold:

(5.8.1) (Cf. [11, Remark 2.23(i)] and [13, Theorem 2.2] .) If $R$ is Henselian and $R$ is $H_{0}, H_{1}, \ldots, H_{a}$, then $R$ is quasi-unmixed (and conversely).

(5.8.2) (Cf. [11, Theorem 2.21] and [13, Theorem 2.2] .) If $R[X]_{(M, X)}$ is $H_{0}, H_{1}, \ldots, H_{a+1}$, then $R$ is quasi-unmixed (and conversely).

Proof. (5.8.1) follows from (5.6.1) $\Longleftrightarrow(5.6 .3)$, and (5.8.2) follows from (5.6.1) $\Longleftrightarrow$ (5.6.2). Q.E.D.

We close this section with two propositions which characterize all local domains $R$ such that card $c(R) \leqslant 2$. For this, we may clearly assume that altitude $R>1$. (In this regard, note that Nagata's examples [6, Example 2, pp. 203-205] have this property.)

(5.9) Proposition. Let $(R, M)$ be a local domain, and let $a=$ altitude $R$ $>$ 1. Then $c(R) \subseteq\{a, n\}(1 \leqslant n \leqslant a)$ if and only if, for each prime ideal $p$ in $R$, if there exists a height one maximal ideal in the integral closure of $R / p$, then height $p \in\{a-1, n-1\}$.

Proof. $c(R) \subseteq\{a, n\}$ if and only if (by (a) $\Rightarrow(b))$ depth $z \in\{a, n\}$, for all minimal prime ideals $z$ in the completion of $R$, if and only if (by (5.2.1) (5.2.4)), for all prime ideals $p$ in $R$, if there exists a height one maximal ideal in the integral closure of $R / p$, then height $p \in\{a-1, n-1\}$. Q.E.D.

To prove the last result of this section, we use the following fact about a catenary local domain $R$ : The integral closure of $R$ satisfies the c.c. if and only if, for each height one prime ideal $p$ in $R, R / p$ satisfies the s.c.c. $[14,(4.3)]$.

(5.10) Proposition. Let $(R, M)$ and $a$ be as in (5.9). Then the following statements hold:

(5.10.1) $c(R)=\{a\}$ if and only if $R$ satisfies the s.c.c. 
(5.10.2) $c(R)=\{a, 1\}$ if and only if $R$ is catenary, $R$ does not satisfy the s.c.c., and the integral closure $R^{\prime}$ of $R$ satisfies the c.c. (2.1.4).

(5.10.3) $c(R)=\{a, n\}(1<n<a)$ if and only if, for all height $h$ prime ideals $p$ in $R(0 \leqslant h \leqslant a), c(R / p) \subseteq\{a-h, n-h\}$ and there exists a prime ideal $q$ in $R$ such that height $q=n-1$ and $1 \in c(R / q)$.

Proof. (5.10.1) is clear from the definitions.

(5.10.2) Assume first that $c(R)=\{a, 1\}$. Then, by (5.6.1) $\Longleftrightarrow(5.6 .7)$, for each prime ideal $p \neq(0)$ in $R, R / p$ is quasi-unmixed (hence $R / p$ satisfies the s.c.c. [10, Theorem 3.1]), and $R$ is $H_{i}(1 \leqslant i \leqslant a)$; hence $R$ is catenary [13, Theorem 2.2$]$. Therefore $R^{\prime}$ satisfies the c.c. $[14,(4.3)]$. Further, by hypothesis, $R$ is not quasi-unmixed, so $R$ does not satisfy the s.c.c.

Conversely, for each height one prime ideal $p$ in $R, R / p$ satisfies the s.c.c. $[14,(4.3)]$ (hence $R / p$ is quasi-unmixed). Therefore, since $S / P$ is quasi-unmixed if $S$ is [6, (34.5)] (where $S$ is a local domain), it follows that, for all nonzero prime ideals $p$ in $R, R / p$ is quasi-unmixed and depth $p=a$ - height $p$ (since $R$ is catenary). Therefore, if $z$ is a minimal prime ideal in the completion of $R$, then depth $z \in\{a, 1\}$, by (5.6.1) $\Longleftrightarrow(5.6 .7)$. Moreover, there exists some $z$ such that depth $z \neq a$, since $R$ is not quasi-unmixed [10, Theorem 3.1]. Hence $c(R)=$ $\{a, 1\}$, by (a) $\Longleftrightarrow(\mathrm{b})$.

(5.10.3) If $c(R)=\{a, n\}$, then for all height $h$ prime ideals $p$ in $R(0 \leqslant$ $h \leqslant a), c(R / p) \subseteq\{a-h, n-h\}[17,(2.25 .1)]$. Also, by (a) $\Longleftrightarrow(\mathrm{b})$, there exists a depth $n$ minimal prime ideal $z$ in the completion $R^{*}$ of $R$, so, by (5.1), there exists a height $n-1$ prime ideal $q$ in $R$ such that there exists a depth one minimal prime ideal in the completion $(R / q)^{*} \cong R^{*} / q R^{*}$ of $R / q$. Therefore $1 \in$ $c(R / q)$, by (a) $\Longleftrightarrow(\mathrm{b})$.

Conversely, if $p$ is a height $h$ prime ideal in $R(0 \leqslant h \leqslant a)$, and if there exists a height one maximal ideal in the integral closure of $R / p$, then $1 \in c(R / p)$ $\subseteq\{a-h, n-h\}$, so $h \in\{a-1, n-1\}$. Therefore, by (5.9), $c(R) \subseteq\{a, n\}$. Finally, since there exists a height $n-1$ prime ideal $q$ in $R$ such that $1 \in c(R / q)$, $n=1+$ height $q \in c(R)[17,(2.25 .1)]$; and clearly $a \in c(R)$. Q.E.D.

It should be noted in (5.10.3) that if $h \geqslant n$, then $c(R / p)=\{a-h\}$; hence $R / p$ satisfies the s.c.c., for all prime ideals $p$ in $R$ such that height $p \geqslant n$. Also, by letting $n=1$ in (5.10.3) we get (5.10.2).

6. Some specific overrings. In this section we construct some finitely-generated overrings of a local domain $R$ (rings which contain $R$ and are contained in the quotient field of $R$ ) which have maximal chains of prime ideals with certain properties. These results could, for the most part, have been given in $\S 2$, but it was felt that the readability of this paper would be improved by delaying the material to the final section of this paper. 
By [3, Theorem 1.10], if $(R, M)$ is a local domain and $n \in c(R)$, then there exists a principal integral extension domain $R[c]$ of $R$ which has a mcpil $n$. The following proposition shows that there exists a locality $A$ of the form $A=$ $R[d]_{(M, d)}$ contained in the quotient field of $R$ which has a mcpil $n$.

(6.1) Proposition. Let $F$ be the quotient field of a local domain $(R, M)$. Then $n \in c(R)$ if and only if there exists a locality $A=R[d]_{(M, d)} \subseteq F(A d e$ pends on $n$ ) which has a mopil $n$.

Proof. Let $n \in c(R)$. Then, by (5.2.1) $\Longleftrightarrow(5.2 .4)$, there exists a prime ideal $p$ in $R$ such that height $p=n-1$ and such that there exists a height one maximal ideal $N$ in the integral closure $S$ of $R / p$. Therefore, if depth $p=1$, then let $d=0$ and $A=R$. If depth $p>1$, then let $x \in N$ such that $x$ is not in any other maximal ideal in $S$. Let $x=c^{\prime} / b^{\prime}$, with $c^{\prime}$ and $b^{\prime}$ in $R / p$, and let $A^{\prime}=$ $(R / p)[x]_{(M / p, x)}$, so altitude $A^{\prime}=1$ (since $S_{N}$ is the integral closure of $\left.A^{\prime}\right)$. Let $b$ and $c$ be preimages in $R$ of $b^{\prime}$ and $c^{\prime}$. Then $(R / p)[x] \cong R[c / b] / p^{*}$, where $p^{*}=p R[1 / b] \cap R[c / b] ;$ hence $A^{\prime} \cong A / p^{*} A$, where $A=R[c / b]_{(M, c / b)}$, and so depth $p^{*} A=1$. Also, height $p^{*} A=$ height $p^{*}=$ height $p=n-1$, so there exists a mcpil $n$ in $A$.

The converse was given by (2.9). Q.E.D.

(6.2) Corollary (CF. [13, Theorem 3.3]). Let $(R, M)$ and $F$ be as in (6.1). Then $R$ satisfies the s.c.c. if and only if, for all localities $A=R[d]_{(M, d)}$ $\subseteq F$, altitude $A=$ altitude $R$ and $A$ is catenary.

Proof. Clear, by (2.4.2) and (6.1). Q.E.D.

In (2.8.2) it was shown, in particular, that if $A$ is a finitely-generated algebraic extension domain of a local domain $R$, and if $N$ is a maximal ideal in $A$, then height $N \in c(R)$. The converse of this will now be given.

(6.3) Proposition. Let $(R, M)$ and $F$ be as in (6.1), and let $n \in c(R)$. Then there exists a finitely-generated ring $A$ over $R$ such that $A \subset F$ and $A$ has a maximal ideal $N$ such that $N \cap R=M$ and height $N=n$.

Proof. By (3.7), let $B$ be an $M$-primary ideal such that there exists a maximal relevant ideal $Q$ in $R=R(R, B)$ such that height $Q=n$. Then, since $Q$ is relevant, $t b \notin Q$, for some $b \in B$. Let $C=R[1 / t b]$ and $A=R[B / b]$. Then $C=A[t b, 1 / t b]$. Also, by $[11, \operatorname{Remark} 3.11], N=Q C \cap A$ is a prime ideal such that $N C \cap R=Q$; hence (since $t b$ is transcendental over $A$ ) height $N=$ height $Q$ $=n$. Further, by $[11$, Remark 3.11], depth $N=\operatorname{depth} Q-1$. Hence, by (3.5.3), $N$ is a maximal ideal in $A$. Finally, $N \cap R=Q \cap R=M$, since $(M, u) R$ $\subseteq Q$ (3.5.3). Q.E.D.

(6.4) RemaRK. Let $(R, M)$ and $B$ be as in (3.7). If $R / M$ is an infinite 
field, then it can be shown that given maximal relevant ideals $Q_{1}, \ldots, Q_{k}$ in $R$ $=R(R, B)$ such that $\left\{\right.$ height $\left.Q_{i} ; i=1, \ldots, k\right\}=c(R)$ (see (3.7)) there exists $b \in B$ such that $t b \notin \bigcup Q_{i}$; hence $A=R[B / b]$ has, by (6.3) and its proof, maximal ideals $P_{i}=Q_{i} C \cap A$ such that $P_{i} \cap R=M$ and $\left\{\right.$ height $P_{i} ; i=1, \ldots$, $k\}=c(R)$.

The next result shows that if $m>1$ is the smallest element in $c(R)$, then there exists a quadratic transformation $L$ of $R$ such that $n \in c(R)$ if and only if $n-m+1 \in c(L)$. To prove this result, we need the following lemma which should be of use in other problems involving the completion of a local ring.

(6.5) Lemma. Let $\left(R^{*}, M^{*}\right)$ be the completion of a local ring $(R, M)$, and let $z_{1}, \ldots, z_{g}$ be minimal prime ideals in $R^{*}$. Assume that $n=$ $\min \left\{\right.$ depth $\left.z_{j} ; j=1, \ldots, g\right\} \geqslant 1$. Then there exist $b_{1}, \ldots, b_{n}$ in $M$ such that, with $B_{j, i}=\left(z_{j}, b_{1}, \ldots, b_{i}\right) R^{*}(j=1, \ldots, g$ and $i=0,1, \ldots, n$ (for $i=0$, $\left.B_{j, 0}=z_{j}\right)$ ) the following statements hold:

(6.5.1) If $p$ is a minimal prime divisor of $B_{j, i}$ and $i<n$, then height $p=$ height $p / z_{j}=i$

(6.5.2) If $p$ is a minimal prime divisor of $B_{j, n}$ and depth $z_{j}>n$, then height $p=$ height $p / z_{j}=n$. If depth $z_{j}=n$, then $B_{j, n}$ is $M^{*}$-primary.

Proof. Assume first that $n>1$ and that $b_{1}, \ldots, b_{k}(0 \leqslant k<n-1)$ are elements in $M$ such that each minimal prime divisor $p$ of each $B_{j, i} \quad(j=1$, $\ldots, g$ and $i=0,1, \ldots, k)$ is such that height $p=$ height $p / z_{j}=i$. Let $S^{\prime}=$ $\left\{q ; q\right.$ is a minimal prime divisor of $B_{j, k}$, for some $\left.j=1, \ldots, g\right\}$ and let $S^{\prime \prime}=$ $\left\{p^{*} \in \operatorname{Spec} R^{*}\right.$; there exists $q \in S^{\prime}$ such that $q \subset p^{*}$, height $p^{*} / q=1$, and height $p^{*}>$ height $q+1=k+1$ \}. Let $S=S^{\prime} \cup S^{\prime \prime}$, so $S$ is a finite set, by [2, Theorem 1]. Also, $M^{*} \notin S$, since $k<n-1$ and each $R^{*} / z_{j}$ is catenary. Therefore, let $b_{k+1} \in M, \notin \bigcup\{P \cap R ; P \in S\}$, and let $Q$ be a minimal prime divisor of $B_{j, k+1}$ (for some $j=1, \ldots, g$ ). Then $Q$ properly contains a minimal prime divisor $q$ of $B_{j, k}$ (since $S^{\prime} \subseteq S$ ), so height $Q \geqslant k+1$. Also, height $Q / q=$ 1 , by the principal ideal theorem, so height $Q / z_{j}=k+1$ (since $R^{*} / z_{j}$ is catenary and height $q / z_{j}=k$ ). Further, height $Q \ngtr k+1$, since $S^{\prime \prime} \subseteq S$. Therefore height $Q=$ height $Q / z_{j}=k+1$. Hence it follows that $b_{1}, \ldots, b_{n-1}$ exist, if $n>1$.

To pick $b_{n}$ (if $n>1$ ) or to pick $b_{1}$ (if $n=1$ ) such that (6.5.2) holds, let $S^{\prime}$ be as above for $k=n-1$. Let $d$ be such that depth $z_{j}=n$ if and only if $j \geqslant d(1 \leqslant d \leqslant g)$. If $d=1$, then any $b_{n} \in M, \notin \bigcup\left\{P \cap R ; P \in S^{\prime}\right\}$ will do, so assume that $d>1$. Let $S^{\prime \prime}=\left\{p^{*} \in \operatorname{Spec} R^{*}\right.$; there exists a minimal prime divisor $q$ of $B_{j, n-1}(j<d)$ such that $q \subset p^{*}$, height $p^{*} / q=1$, and height $p^{*}$ $>n$ \}. Let $S=S^{\prime} \cup S^{\prime \prime}$, so $S$ is a finite set and $M^{*} \notin S$. Let $b_{n} \in M, \notin$ $\bigcup\{P \cap R ; P \in S\}$. Then it is seen as above that, for each minimal prime divisor $Q$ of $B_{j, n}(j<d)$, height $Q=$ height $Q / z_{j}=n$; and $B_{j, n}$ is $M^{*}$-primary, for $j=$ $d, \ldots, g$. Q.E.D. 
(6.6) REMARK. With the notation of (6.5), if $z_{1}, \ldots, z_{g}$ are all the minimal prime ideals in $R^{*}$, then height $\left(b_{1}, \ldots, b_{i}\right) R=$ height $\left(b_{1}, \ldots, b_{i}\right) R^{*}$ $=i$, for $i=1, \ldots, n$.

Proof. Let $B_{i}=\left(b_{1}, \ldots, b_{i}\right) R$ and suppose that height $B_{i}<i$, for some $i$. Then height $B_{i} R^{*}=$ height $B_{i}<i$, so there exists a minimal prime divisor $p^{*}$ of $B_{i} R^{*}$ such that height $p^{*}<i$. Then $z_{j} \subseteq p^{*}$, for some $j=1, \ldots, g$, so height $\left(z_{j}, B_{i}\right) R^{*}<i$; contradiction (6.5). Therefore height $B_{i}=$ height $B_{i} R^{*}$ $=i(i=1, \ldots, n)$. Q.E.D.

The following result shows that the converse of (2.10) is sometimes true. It also shows that the condition that $R$ be a subspace of $L$ in (3.1) is stronger than is needed for its conclusion.

(6.7) Proposition. Let $(R, M)$ be a local domain, and assume that $\min \left\{\right.$ depth $z ; z$ is a minimal prime ideal in the completion $R^{*}$ of $\left.R\right\}=m>1$. Then, for each $k=2, \ldots, m$, there exist analytically independent elements $b_{1}, \ldots, b_{k}$ in $R$ such that, with $A=R\left[b_{2} / b_{1}, \ldots, b_{k} / b_{1}\right], n \in c(R)$ if and only if $n-k+1 \in c\left(A_{M A}\right)$.

Proof. Let $z_{1}, \ldots, z_{g}$ be the minimal prime ideals in $R^{*}$, and let $b_{1}$, $\ldots, b_{k}(2 \leqslant k \leqslant m)$ be as in (6.5). Let $B=\left(b_{1}, \ldots, b_{k}\right) R$. Then height $B=$ $k$ (6.6), so the $b_{i}$ are a subset of a system of parameters in $R$; hence they are analytically independent in $R$. Therefore, with $A$ as above, $M A$ is a prime ideal such that height $M A=$ height $M-(k-1)$ and depth $M A=k-1$ [11, Lemma 4.3]. Let $D=R^{*}\left[b_{2} / b_{1}, \ldots, b_{k} / b_{1}\right]$, fix $j$ and let $z=z_{j}$, and let $w=$ $z R^{*}\left[1 / b_{1}\right] \cap D$. Then, since $b_{1}+z, \ldots, b_{k}+z$ are a subset of a system of parameters in $R^{*} / z$ (6.5), $w \subset M^{*} D\left[11\right.$, Remark 4.4(i)] and height $M^{*} D / w=$ height $M^{*} / z-(k-1)$ [11, Lemma 4.3]. Therefore depth $w D_{M^{*} D}=$ depth $z-k+1$. Also, $A_{M A}$ is a dense subspace of $D_{M^{*} D}$ [11, Lemma 3.2], so, as in the proof of (3.1) and since $z=z_{j}$ was arbitrary, \{depth $q ; q$ is a minimal prime ideal in the completion of $\left.A_{M A}\right\}=\left\{\right.$ depth $\left.z_{j}-k+1 ; j=1, \ldots, g\right\}$. Therefore, by (a) $\Longleftrightarrow$ (b), $n \in c(R)$ if and only if $n-k+1 \in c\left(A_{M A}\right)(k=$ $2, \ldots, m)$. Q.E.D.

(6.8) REMARK. It is clear from the proof of (6.7) that, if $z_{1}, \ldots, z_{g}$ are minimal prime ideals in the completion of $R$ (possibly not all of them) and $d_{t}=$ depth $z_{t}>1(i=1, \ldots, g)$, then there exist analytically independent elements $b_{1}, \ldots, b_{m}\left(m=\min d_{i}\right)$ in $R$ such that, for $k=2, \ldots, m, d_{i}-k+1$ $\in c\left(A_{M A}\right)(i=1, \ldots, g)$, where $A=R\left[b_{2} / b_{1}, \ldots, b_{k} / b_{1}\right]$.

This paper will be closed with the following remark. The remark stands in relation to (6.7) much as (6.2) stands in relation to (6.1).

(6.9) Remark. Let $(R, M)$ be a local domain, and let $a=$ altitude $R$. 
Then the following statements hold:

(6.9.1) If $R$ is catenary, then, for all analytically independent elements $b$, $c$ in $R, R(c / b)=R[c / b]_{M R[c / b]}$ is catenary and altitude $R(c / b)=a-1$ (and conversely) [12, Theorem 4.12].

(6.9.2) If, for all analytically independent elements $b, c$ in $R, R(c / b)$ satisfies the s.c.c. and altitude $R(c / b)=a-1$, then either $R$ satisfies the s.c.c. or $R$ is catenary and the integral closure $R^{\prime}$ of $R$ satisfies the c.c.

Proof. (6.9.2) Let $z$ be a minimal prime ideal in the completion $R^{*}$ of $R$. If $n=\operatorname{depth} z>1$, then there exist analytically independent elements $b, c$ in $R$ such that $n-1 \in c(R(c / b))(6.8)$. Therefore, by hypothesis, $n-1=$ altitude $R(c / b)=a-1$; hence $n=a$. Thus, for all minimal prime ideals $z$ in $R^{*}$, depth $z \in\{1, a\}$, so the conclusion follows from (5.10.2). Q.E.D.

\section{BIBLIOGRAPHY}

1. S. Abhyankar, Resolution of singularities of embedded algebraic surfaces, Pure and Appl. Math., vol. 24, Academic Press, New York and London, 1966. MR 36 \#164; erratum, 36, p. 1568.

2. S. McAdam, Saturated chains in Noetherian rings, Indiana Univ. Math. J. 23 (1973/74), 719-728. MR 48 \#11094.

3. S. McAdam and E. G. Houston, Chains of primes in Noetherian rings, Indiana Univ. Math. J. 24 (1975), 741-753.

4. S. McAdam and L. J. Ratliff, Jr., Semi-local taut rings (forthcoming).

5. M. Nagata, On the chain problem of prime ideals, Nagoya Math. J. 10 (1956), 5164. MR 18, 8 .

6. - Local rings, Interscience Tracts in Pure and Appl. Math., no. 13, Interscience, New York, 1962. MR 27 \#5790. side, 1973.

7. M. E. Pettit, Jr., Properties of $H_{i}$-rings, Ph. D. Thesis, University of California, River-

8. M. E. Pettit, Jr., Properties of $H_{i}$-rings (forthcoming).

9. L. J. Ratliff, Jr., On quasi-unmixed semi-local rings and the altitude formula, Amer. J. Math. 87 (1965), 278-284. MR 31 \#3448.

10. - On quasi-unmixed local domains, the altitude formula, and the chain condition for prime ideals. I, Amer. J. Math. 91 (1969), 508-528. MR 40 \#136.

11. - On quasi-unmixed local domains, the altitude formula, and the chain condition for prime ideals. II, Amer. J. Math. 92 (1970), 99-144. MR 42 \#249.

12. - Characterizations of catenary rings, Amer. J. Math. 93 (1971), 10701108. MR 45 \#6804.

13. - Catenary rings and the altitude formula, Amer. J. Math. 94 (1972), 458-466. MR 47 \#221.

14. - Chain conjectures and H-domains, Conf. on Commutative Algebra (Univ. Kansas, Lawrence, Kan., 1972), Lecture Notes in Math., vol. 311, Springer-Verlag, Berlin, 1973. MR 49 \#2714.

15. - Four notes on saturated chains of prime ideals, J. Algebra 39 (1976), 75-92.

16. Equivalences of the chain conjectures (forthcoming).

17. L. J. Ratliff, Jr. and S. McAdam, Maximal chains of prime ideals in integral extension domains. I, Trans. Amer. Math. Soc. 224 (1976), 103-116.

18. L. J. Ratliff, Jr. and M. E. Pettit, Jr., Characterizations of $H_{i}$-local rings and of $C_{i}$-local rings (forthcoming). 
19. D. Rees, $A$ note on form rings and ideals, Mathematika 4 (1957), 51-60. MR 19, 835.

20. - A-transforms of local rings and a theorem on multiplicities of ideals, Proc. Cambridge Philos. Soc. 57 (1961), 8-17. MR 22 \#9521.

21. O. Zariski and P. Samuel, Commutative algebra. Vol. II, University Ser. in Higher Math., Van Nostrand, Princeton, N. J., 1960. MR 22 \#11006.

DEPARTMENT OF MATHEMATICS, UNIVERSITY OF CALIFORNIA, RIVERSIDE, CALIFORNIA 92502 